\title{
Good's syndrome with a relapsed bloodstream infection induced by Alcaligenes sp. after thymectomy: A case report
}

\author{
SUMEI CHEN", JIANGFU LIU*, YONGNIAN LIN and YIPENG GAO \\ Department of Infectious Diseases, The First Hospital of Quanzhou Affiliated to Fujian Medical University, \\ Quanzhou, Fujian 362000, P.R. China
}

Received October 31, 2019; Accepted December 14, 2020

DOI: $10.3892 /$ etm.2021.9830

\begin{abstract}
Good's syndrome (GS) or thymoma-associated immunodeficiency is a rare clinical syndrome with poor prognosis. The varied and unspecified clinical manifestations of GS commonly lead to a missed or delayed diagnosis. Thus, misdiagnosis and missed diagnosis are common. The present case study reports on a patient who suffered from a relapsed bloodstream infection caused by Alcaligenes sp. 6 months after thymectomy. The patient was finally diagnosed with GS after analyzing the clinical features and detecting T-lymphocyte subsets in the peripheral blood and humoral immune function. The patient's condition improved after anti-infection treatment and supplementation with intravenous immunoglobulin. Furthermore, no infection was observed during the 1-year follow-up.
\end{abstract}

\section{Introduction}

Good's syndrome (GS), also known as thymoma-associated immune deficiency syndrome, is a rare primary immune deficiency disease that occurs in individuals aged 40-60 years. Common clinical features are thymoma, hypogammaglobulinemia, low peripheral B-lymphocyte count and inverted $\mathrm{CD}^{+} / \mathrm{CD}^{+} \mathrm{T}$-cell ratio. Thymoma is the most common tumor type in the anterior mediastinum and most thymomas may cause immune dysfunction. Furthermore, 5-10\% of patients with thymoma have increased susceptibility to bacterial and opportunistic viral and fungal infections (1-3). In 1954, this

Correspondence to: Dr Sumei Chen, Department of Infectious Diseases, The First Hospital of Quanzhou Affiliated to Fujian Medical University, 250 East Street, Licheng, Quanzhou, Fujian 362000, P.R. China

E-mail: chensm561119190@sina.com

*Contributed equally

Abbreviations: GS, Good's syndrome.

Key words: Alcaligenes sp., hypogammaglobulinemia, thymoma, Good's syndrome phenomenon was first described by Dr Robert Good (4). Later, immunological studies demonstrated that most patients also had hypogammaglobulinemia, B-cell reduction or deficiency and inversion of the CD4/CD8 ratio, which now part of the definition of Good's syndrome. A prominent feature of patients with GS is loss of B cells. Furthermore, the prognosis is poor and mortality is high. Infections are the most common cause of death $(5,6)$. However, Thongngarm et al (7) examined the clinical course and outcomes for nine patients with GS after treatment with intravenous immunoglobulin at Thailand's largest national tertiary referral hospital. They reported that seven patients had favorable clinical outcomes.

\section{Case report}

Written informed consent was obtained from the patient for the publication of this case report and accompanying images. The patient was an unemployed 42-year-old Han Chinese male. In summary, the patient underwent thymectomy and after 6 months, a relapsed respiratory tract infection and was diagnosed with Alcaligenes sp. infection as detected by three blood cultures. After analysis of the clinical and immunological characteristics, the patient was finally diagnosed with GS. The patient's condition was stable after receiving anti-infection treatment and regular supplementation with gamma globulin. This was the second reported case of GS combined with Alcaligenes sp. to date. A detailed case description is provided below.

The patient had a fever for 3 days. The body temperature fluctuated between 36.5 and $38.5^{\circ} \mathrm{C}$, accompanied by throat discomfort, cough, phlegm and muscle soreness. The patient was admitted to the Infectious Disease Unit of the First Hospital of Quanzhou Affiliated to Fujian Medical University (Quanzhou, China) in November 2017.

In May 2017, the patient underwent enhanced CT of the lungs due to cough for $>1$ month (Fig. 1), which revealed occupying lesions in the left anterior mediastinum, slightly enlarged lymph nodes and two hilus pulmonis. Therefore, thymectomy was performed the First Hospital of Quanzhou Affiliated to Fujian Medical University (Quanzhou, China). The post-operative pathological diagnosis was type AB thymoma (Fig. 2). The patient had a severe infection after the surgery and recovered after anti-infection treatment and supplementation with gamma globulin. In June 2017, humoral 
immune function suggested that immunoglobulin ( $\mathrm{Ig}) \mathrm{G}, \mathrm{IgM}$ and IgA levels were markedly reduced (Table I), which was left untreated. The patient was previously healthy and none of his family members had any similar symptoms. The patient had no history of smoking, drinking or infectious diseases.

Physical examination on admission revealed the following: The body temperature was $37.8^{\circ} \mathrm{C}$. The patient was in a good mental state and had a low BMI of 17.5. He had pharyngeal congestion but no rales in the lungs. The auxiliary examination after admission revealed the following: Among the routine blood parameters (8), the leukocyte count increased $\left(13.10 \times 10^{9} / 1\right.$, normal range: $\left.3.5-9.5 \times 10^{9} / 1\right)$, the neutrophil percentage increased $(76.40 \%$, normal range $40-75 \%)$, the lymphocyte count was normal $\left(2.06 \times 10^{9} / 1\right.$, normal range: $\left.1.1-3.2 \times 10^{9} / 1\right)$, the erythrocyte count was normal $(4.99 \mathrm{~g} / 1$ $\mathrm{x} 10^{12} / 1$, normal range: $\left.4.3-5.8 \times 10^{12} / 1\right)$, hemoglobin was normal (142 g/l, normal range: 130-175 g/l) and the platelet count was normal $\left(305 \times 10^{9} / 1\right.$, normal range: $\left.125-350 \times 10^{9} / 1\right)$. The C-reactive protein level increased (148.45 mg/l, normal range $<8 \mathrm{mg} / \mathrm{l})$. In regards to the humoral immune function, $\mathrm{IgG}, \operatorname{IgM}$ and $\operatorname{Ig}$ A levels were markedly decreased (Table I). The pulmonary CT scan revealed changes to the postoperative thymoma and inflammation in the left lower lung after thymectomy. The bilateral double-tube blood culture indicated the presence of Alcaligenes sp. [blood culture and identification method: Specimens were cultured in an FX400 blood culture instrument (BD Biosciences), positive specimens were transferred to the blood plate and strains were identified using a Phoenix 100 automatic bacterial identification system and its supporting bacteria identification card (BD Biosciences)]. The strains were susceptible to piperacillin and resistant to cefotaxime and levofloxacin. Anti-infection treatment using ticarcillin-clavulanate potassium (intravenous infusion at $3.2 \mathrm{~g} \mathrm{q} 6 \mathrm{~h}$ ) was performed for 1 week. After 1 day of infusion the body temperature decreased to normal. After 1 week, the cough and phlegm improved and blood culture was negative upon reanalysis. Hene, the patient was discharged. The patient received sequential oral amoxicillin (1.0 bid for 7 days) and he discontinued the medication voluntarily after 3 days.

At two weeks after discharge, the patient was readmitted to the hospital due to fever (maximum temperature, $38.2^{\circ} \mathrm{C}$ ), cough and phlegm. After admission, the bilateral double-tube blood culture revealed Alcaligenes sp. (the methods of cultivation and identification were the same as those described above). A pulmonary CT scan indicated an improvement in the inflammation of the left lower lobe. Furthermore, anti-infection treatment with the same medicines (ticarcillin-clavulanate potassium intravenous infusion at $3.2 \mathrm{~g} \mathrm{q} 6 \mathrm{~h}$ ) was performed and the course of the treatment was prolonged to 2 weeks. The body temperature decreased to normal following 1 day of infusion. The blood culture was negative and hence, the patient was discharged. Sulfamethoxazole tablets ( 2 tablets bid for 7 days) were orally administered sequentially.

At two weeks after discharge, the patient had a fever with a temperature of $37.8^{\circ} \mathrm{C}$, a sore throat and a mild cough. Hence, he was admitted for the third time. A similar blood culture confirmed the presence of Alcaligenes sp. (the methods of cultivation and identification were the same as those described earlier). An expanded examination was performed to further detect any potential lesions. No significant abnormality was observed by transthoracic echocardiography and enhanced CT. Electronic gastroscopy and colonoscopy indicated fungal esophagitis. The humoral immunoassay indicated a decrease in IgG, IgM and IgA levels (Table I). Analysis of T-lymphocyte subsets suggested a decrease in the numbers of B and $\mathrm{CD} 4^{+}$ $\mathrm{T}$ cells and the CD4/CD8 ratio, while the number of $\mathrm{CD}^{+}$ $\mathrm{T}$ cells was increased (Table II). Thus, besides the previous thymoma, recurrent bloodstream infection and B-cell deficiency, $\mathrm{CD}^{+}{ }^{+} \mathrm{T}$-cell reduction, $\mathrm{CD}^{+} \mathrm{T}$-cell elevation, inversion of CD4/CD8 ratio and hypogammaglobulinemia had been confirmed by a peripheral blood test. The patient was finally diagnosed with GS. He was diagnosed with a secondary (secondary to a respiratory tract infection) recurrent bloodstream infection (Alcaligenes sp.), respiratory infection and fungal esophagitis. The same medications as those received previously (ticarcillin-clavulanate potassium intravenous infusion at $3.2 \mathrm{~g} \mathrm{q} 6 \mathrm{~h}$ ) were administered for 2 weeks, followed by intravenous supplementation with gamma globulin $(5 \mathrm{~g} / \mathrm{d}$ for 7 days) and subcutaneous injection of thymalfasin $(1.6 \mu \mathrm{g}$ q3d) simultaneously to improve immunity. Furthermore, antifungal treatment was performed by oral administration of fluconazole ( $300 \mathrm{mg}$ qd for 14 days). The patient understood and accepted the symptoms of GS and compliance was good. After discharge, the patient voluntarily infused gamma globulin at $5 \mathrm{~g} / \mathrm{d}$ for 2 days and thymalfasin (1.6 $\mu \mathrm{g} \mathrm{q3d}$ ) was subcutaneously injected in an outpatient setting. The patient was followed up once every 1-2 months and no infection developed within 1 year. However, hypogammaglobulinemia and decreased $\mathrm{CD}^{+}{ }^{+} \mathrm{T}$ cells persisted (Table I).

\section{Discussion}

GS is an adult-onset T-cell and B-cell combined immunodeficiency, which is associated with thymoma, manifesting as recurrent infection, paraneoplastic syndrome and autoimmune disease $(9,10)$. Its clinical manifestations are diverse. It has no specificity, irrespective of symptoms associated with thymoma or infections caused by immune deficiency. Early diagnosis is difficult, besides the insufficient awareness of clinicians. A meta-analysis performed by Dong et al (3) in China indicated that the time interval between symptom onset and the diagnosis of GS ranged from 5 days to 11 years. The humoral immunity of the patient of the present study was significantly lower at the time-point of surgery. A severe infection occurred after the surgery, but it was not recognized as GS. The disease remained undetected until the occurrence of the recurrent bloodstream infection caused by Alcaligenes sp. the third time 6 months later, as no difficult-to-clear primary or migratory lesions were observed during a comprehensive examination. The observations suggested that hypogammaglobulinemia may provide a good clue for clinical diagnosis when treating patients with a previous history of thymoma combined with recurrent infections.

Infections are the most common clinical manifestation and cause of death in patients with GS, particularly respiratory, skin and intestinal infections $(1,3,6)$. A previous study demonstrated that the degree of B-cell and T-cell dysfunction and hypogammaglobulinemia were the most important factors affecting the intensity of infection (9). In the present case, the humoral immunity of peripheral blood 


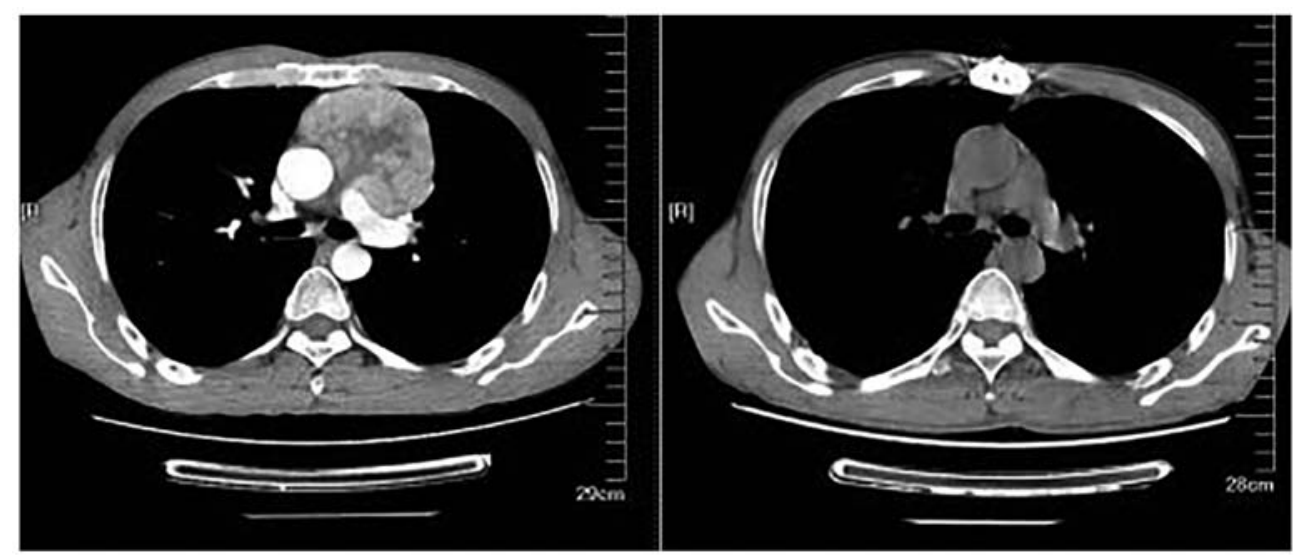

Figure 1. Mediastinal window on pulmonary CT. The left image was acquired May 2017 prior to the surgery and the right image was acquired in November 2017, after the surgery.

A

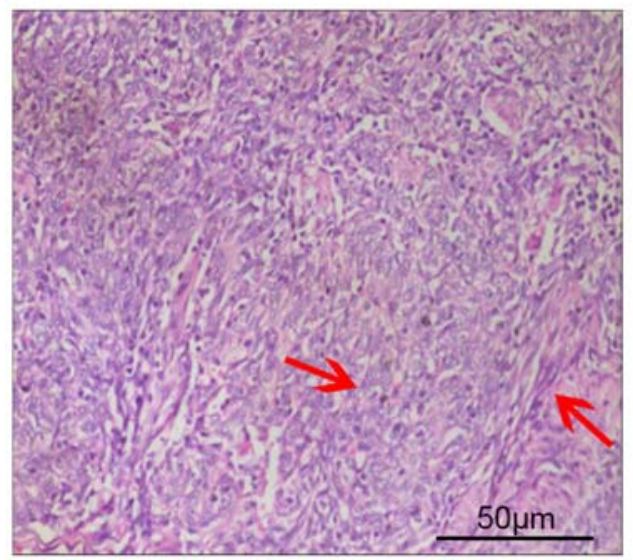

C

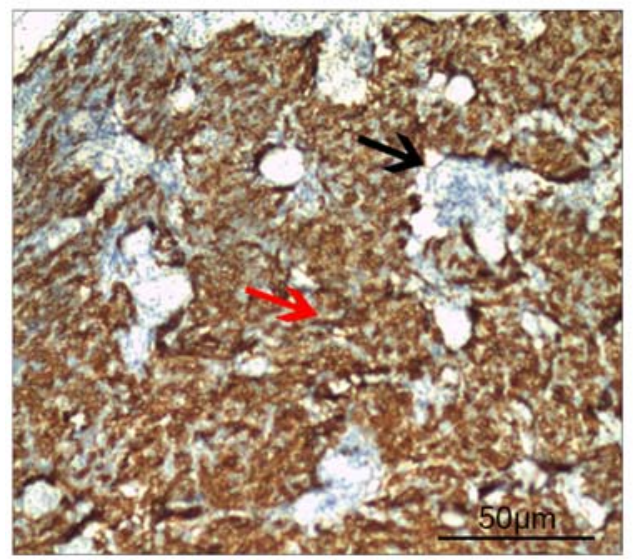

B

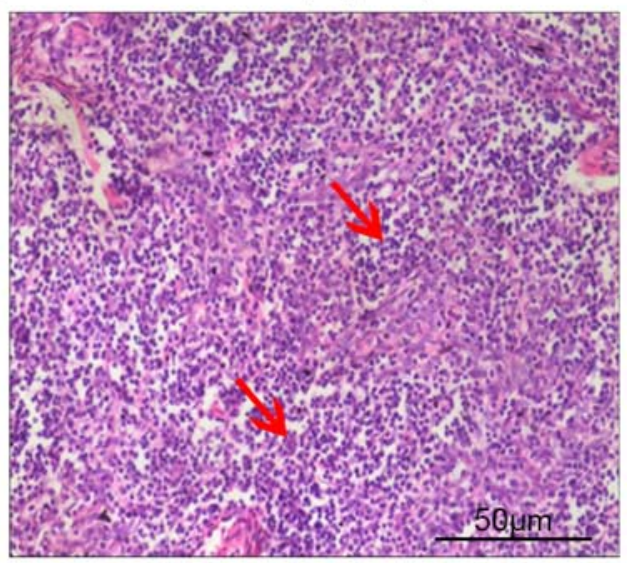

D

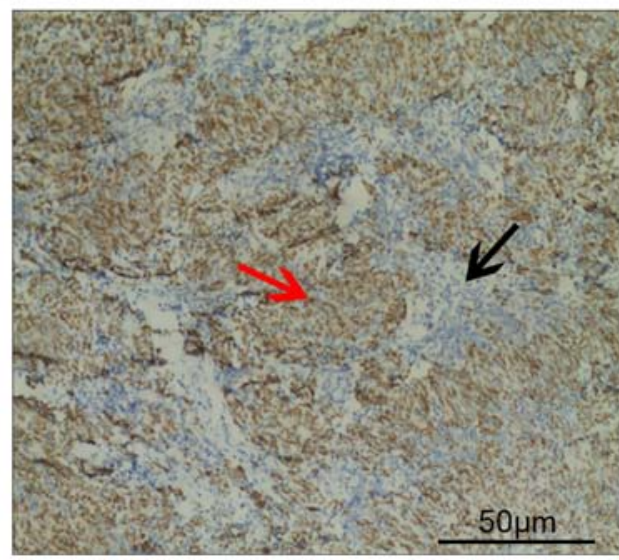

Figure 2. Pathology: Thymoma (AB type). (A) H\&E staining indicated tumor cells presented a spindle-like morphology. (B) H\&E staining displaying mixed lymphocytes of tumor cells. Immunohistochemical analysis revealing (C) CK19 (+) status and (D) P63 (+) status with positive staining displaying as orange-yellow. In C and D, the red arrows indicate positive cells and the black arrows indicate negative cells (scale bars, $50 \mu \mathrm{m}$ ).

was tested four times and the T-cell subset was tested once. The results suggested that the total B-cell $\left(\mathrm{CD} 19^{+}\right)$count was significantly decreased (1.6 cells/ $\mu \mathrm{l})$. In addition, $\mathrm{IgG}$ fluctuated between 1.66 and $2.08 \mathrm{~g} / 1$ without an infusion of $\mathrm{Ig}, \mathrm{IgA}$ fluctuated between 0.09 and $0.14 \mathrm{~g} / \mathrm{l}$ and $\mathrm{IgM}$ was $<0.046 \mathrm{~g} / 1$. Furthermore, the $\mathrm{CD} 3^{+} \mathrm{CD} 4^{+} \mathrm{T}$-lymphocyte count was decreased $(336$ cells/ $\mu \mathrm{l})$ and the CD4/CD8 ratio was inverted (0.17). However, the $\mathrm{CD}^{+} \mathrm{CD} 8^{+} \mathrm{T}$-cell count was significantly increased $(2,018$ cells $/ \mu 1)$. These results were consistent with those of a meta-analysis by Chen et al (11), which also indicated that the numbers of different types of regulatory $\mathrm{T}$ cells in the peripheral blood were higher compared with those in healthy controls. The distribution of immune cells in the peripheral blood was different from that in 
Table I. Results of multiple immune tests and intervention measures at different time-points.

\begin{tabular}{|c|c|c|c|c|}
\hline Time-point & $\begin{array}{c}\mathrm{IgG}, \mathrm{g} / \mathrm{l} \\
(\mathrm{RR}, 7.51-15.6)\end{array}$ & $\begin{array}{c}\text { IgM, g/l } \\
(\mathrm{RR}, 0.46-3.04)\end{array}$ & $\begin{array}{c}\text { IgA, g/l } \\
(\mathrm{RR}, 0.82-4.53)\end{array}$ & Intervention measures \\
\hline June 2017 & 1.66 & $<0.0417$ & 0.0938 & $\begin{array}{l}20 \mathrm{~d} \text { after thymoma resection, intravenous } \\
\text { supplementation with gamma globulin at } 5.0 \mathrm{~g} \\
\text { was performed once }\end{array}$ \\
\hline November 2017 & 2.08 & $<0.0417$ & 0.103 & Gamma globulin was not infused \\
\hline January 2018 & 10.2 & 0.0470 & 0.140 & $\begin{array}{l}\text { Intravenous supplementation of gamma globulin } \\
(5 \mathrm{~g} / \mathrm{d} \text { for } 7 \mathrm{~d}) \text { and subcutaneous injection of } \\
\text { thymalfasin }(1.6 \mu \mathrm{g} \mathrm{q} 3 \mathrm{~d})\end{array}$ \\
\hline February 2018 & 6.55 & 0.14 & 0.141 & $\begin{array}{l}\text { Intravenous supplementation of gamma globulin } \\
(5 \mathrm{~g} / \mathrm{d} \text { for } 2 \mathrm{~d}) \text { and subcutaneous injection of } \\
\text { thymalfasin }(1.6 \mu \mathrm{g} \mathrm{q} 3 \mathrm{~d})\end{array}$ \\
\hline
\end{tabular}

RR, reference range; d, days.

Table II. Results for lymphocyte subsets (7 months after thymoma operation).

\begin{tabular}{llcc}
\hline Item & Percentage (RR) & Concentration, cells/ $\mu$ l or ratio (RR) & Trend \\
\hline Total T cells $\left(\mathrm{CD}^{+}\right)$ & $90.28(60.8-80.1)$ & $2,421.08(937.6-1,717.3)$ & $\uparrow$ \\
$\mathrm{CD}^{+}$T cells & $13.90(44-66.9)$ & $336.62(518.8-963.9)$ & $\downarrow$ \\
$\mathrm{CD} 8^{+}$T cells & $83.36(25.9-47.2)$ & $2,018.11(326.7-640.2)$ & $\uparrow$ \\
$\mathrm{CD} 4 / \mathrm{CD} 8\left(\mathrm{CD} 3^{+} \mathrm{CD} 4^{+} / \mathrm{CD}^{+} \mathrm{CD} 8^{+}\right)$ & & $0.17(1.01-2.64)$ & $\downarrow$ \\
Total B cells $\left(\mathrm{CD} 19^{+}\right)$ & $0.06(7.3-19)$ & $1.62(140.6-402.3)$ & $\downarrow$ \\
NK cells $\left(\mathrm{CD} 3{ }^{-} \mathrm{CD} 16^{+} \mathrm{CD} 56^{+}\right)$ & $9.66(7.1-24.5)$ & $258.94(112.1-481.6)$ & \\
\hline
\end{tabular}

NK, natural killer; RR, reference range.

healthy individuals, suggesting that patients with GS are in an immunosuppressed state. However, the intrinsic mechanism underlying the progression of GS should be further investigated.

GS appears to be more complex compared with other immunodeficiency diseases due to the combined immunodeficiency of T cells and B cells. Patients frequently have an infection caused by capsular bacteria. The patient in the present study had an Alcaligenes sp. infection. Alcaligenes widely exists in nature and is a normal constituent of the intestinal flora of humans and animals. Alcaligenes also exists in the respiratory tract, skin and water, and may lead to opportunistic infections. The patient in the present study had pharyngeal discomfort, cough and pharyngeal congestion prior to the onset of the disease; it was considered to be a pulmonary invasion of blood flow. Stark (12) reported on the first case of one patient with GS who had recurrent septicemia caused by alkaline oxidizing xylose due to an indwelling venous catheter. The patient also had osteomyelitis of the fibula, suggesting that the presence of opportunistic pathogens, besides common capsular bacteria, in patients with GS should not be ignored.

At present, no specific treatment for GS is available. Thymectomy may prevent local invasive growth and metastasis of thymoma but it does not improve hypogammaglobulinemia and B-cell deficiency $(13,14)$, which even leads to the deterioration of immune function in certain cases (15). However, supplementation with Igs to maintain serum $\operatorname{IgG}$ at an appropriate concentration and hence reduce infection is currently the most effective treatment for GS. In the present study, the disease recurred after the first antibiotic treatment in hospital, it may be related to an inadequate course of treatment, but the recurrence still occurred after the second hospitalization. Gamma globulin was supplemented after the patient was diagnosed with GS. The patient's condition was then stable with no further recurrence. In a short duration (2 months), the same pathogenic bacteria (Acaligens sp.) invaded three times, indicating that the potential lesions in the patient were not eradicated. It was further confirmed that anti-infection treatment was not sufficient for treating GS; regular supplementation with gamma globulin was required to maintain the IgG/IgM level and hence reduce the occurrence of opportunistic infections. Thongngarm et al (7) suggested that early diagnosis and prompt gamma globulin replacement may change the natural course of this condition and may be successful in keeping patients infection-free. However, it is still necessary to pay attention to intracellular infection and tumor occurrence due to cellular immunodeficiency.

The prognosis of GS is poor. The 5- and 10-year survival rates are 70 and $33 \%$, respectively. The prognosis depends mainly on the severity of relevant infectious diseases, blood 
system diseases and autoimmune diseases and not on the behavior of thymoma $(1,3,16)$. Therefore, follow-up should be strengthened to actively prevent infection.

In conclusion, GS should be alerted for adult patients with a previous history of thymoma and recurrent infections, particularly patients with an opportunistic pathogen infection. Furthermore, humoral immunity and cellular immune function indicators should be carefully screened to facilitate early diagnosis and treatment. For patients diagnosed with GS combined with infection, the treatments are mainly surgical resection of thymoma, Ig replacement therapy and anti-infection treatment.

\section{Acknowledgements}

Not applicable.

\section{Funding}

No funding was received.

\section{Availability of data and materials}

The datasets used and/or analyzed during the present study are available from the corresponding author on reasonable request.

\section{Authors' contributions}

SC performed the examination, treatment and data collection of the patient, as well as the conception, design, drafting and revising of the manuscript. JL participated in data collection and analysis, as well as the design, drafting and revision of the manuscript. YL and YG helped to collect patients' data and revise the manuscript. All authors read and approved the final manuscript.

\section{Ethics approval and consent to participate}

Not applicable.

\section{Patient consent for publication}

Written informed consent was obtained from the patient for publication of this case report and accompanying images.

\section{Competing interests}

The authors declare that they have no competing interests.

\section{References}

1. Kelesidis T and Yang O: Good's syndrome remains a mystery after 55 years: A systematic review of the scientific evidence. Clin Immunol 135: 347-363, 2010.
2. Tarr PE, Sneller MC, Mechanic LJ, Economides A, Eger CM, Strober W, Cunningham-Rundles C and Lucey DR: Infections in patients with immunodeficiency with thymoma (Good syndrome). Report of 5 cases and review of the literature. Medicine (Baltimore) 80: 123-133, 2001.

3. Dong JP, Gao W, Teng GG, Tian Y and Wang HH: Characteristics of Good's Syndrome in China: A systematic review. Chin Med J (Engl) 130: 1604-1609, 2017.

4. Good RA: Absence of plasma cells from bone marrow and lymph nodes following antigenic stimulation in patients with a gamma globulinemia. Rev Hematol 9: 502-503, 1954.

5. Zaman M, Huissoon A, Buckland M, Patel S, Alachkar H, Edgar JD, Thomas M, Arumugakani G, Baxendale H, Burns S, et al: Clinical and laboratory features of seventy-eight UK patients with Good's syndrome (thymoma and hypogammaglobulinaemia). Clin Exp Immunol 195: $132-138,2019$

6. Joven MH, Palalay MP and Sonido CY: Case report and literature review on Good's syndrome, a form of acquired immunodeficiency associated with thymomas. Hawaii J Med Public Health 72: 56-62, 2013

7. Thongngarm T, Boonyasiri A, Pradubpongsa $P$, Tesavibul N, Anekpuritanang $T$, Kreetapirom $P$ and Sompornrattanaphan M: Features and outcomes of immunoglobulin therapy in patients with Good syndrome at Thailand's largest tertiary referral hospital. Asian Pac J Allergy Immunol 37: 109-115, 2019.

8. National Health Commission of the People's Republic of China. Available from: http://www.nhc.gov.cn/wjw/s9492/201110/53169. shtml.

9. Tavakol M, Mahdaviani SA, Ghaemi MR, Vaezi M, Dorudinia A, Jamaati $\mathrm{H}$ and Velayati AA: Good's Syndrome-association of the late onset combined immunodeficiency with thymoma: Review of literature and case report. Iran J Allergy Asthma Immunol 17: 85-93, 2018.

10. Bernard C, Frih H, Pasquet F, Kerever S, Jamilloux Y, Tronc F, Guibert B, Isaac S, Devouassoux M, Chalabreysse L, et al: Thymoma associated with autoimmune diseases: 85 cases and literature review. Autoimmun Rev 15: 82-92, 2016.

11. Chen X, Zhang JX, Shang WW, Xie WP, Jin SX and Wang F: Aberrant peripheral immune function in a good syndrome patient. J Immunol Res 2018: 6212410, 2018.

12. Stark JJ: Alcaligenes xylosoxidans osteomyelitis without trauma in a patient with Good's syndrome. Eur J Intern Med 18: 447, 2007.

13. Kaufman AJ, Palatt J, Sivak M, Raimondi P, Lee DS, Wolf A, Lajam F, Bhora F and Flores RM: Thymectomy for myasthenia gravis: Complete stable remission and associated prognostic factors in over 1000 cases. Semin Thorac Cardiovasc Surg 28: 561-568, 2016.

14. Idress A and Wahla AS: Good syndrome, a rare disease that physicians cannot afford to overlook; case report and review of literature. J Pak Med Assoc 63: 1541-1543, 2013.

15. Ohuchi M, Inoue S, Hanaoka J, Igarashi T, Tezuka N, Ozaki Y and Teramoto K: Good syndrome coexisting with leukopenia. Ann Thorac Surg 84: 2095-2097, 2007.

16. Kelleher P and Misbah SA: What is Good's syndrome? Immunological abnormalities in patients with thymoma. J Clin Pathol 56: 12-16, 2003.

This work is licensed under a Creative Commons Attribution-NonCommercial-NoDerivatives 4.0 International (CC BY-NC-ND 4.0) License. 